\title{
Operando Direct Observation of Filament Formation in Resistive Switching Devices Enabled by a Topological Transformation Molecule
}

Kunqi Hou ${ }^{1}$, Shuai Chen ${ }^{2}$, Cheng Zhou ${ }^{3}$, Linh Lan Nguyen ${ }^{4}$, Putu Andhita Dananjaya ${ }^{1}$, Martial Duchamp ${ }^{4}$, Guillermo C. Bazan ${ }^{3}$, Wen Siang Lew ${ }^{1 *}$ and Wei Lin Leong2*

${ }^{1}$ School of Physical and Mathematical Sciences, Nanyang Technological University 21 Nanyang Link, 637371 Singapore.E-mail: wensiang@ntu.edu.sg

${ }^{2}$ School of Electrical and Electronic Engineering, Nanyang Technological University 50 Nanyang Avenue, 639798 Singapore.E-mail: wlleong@ntu.edu.sg

${ }^{3}$ Depertment of Chemistry, National University of Singapore 3 Science Drive 3, 117543

Singapore

${ }^{4}$ School of Materials Science \& Engineering, Nanyang Technological University 50 Nanyang Avenue, 639798 Singapore 

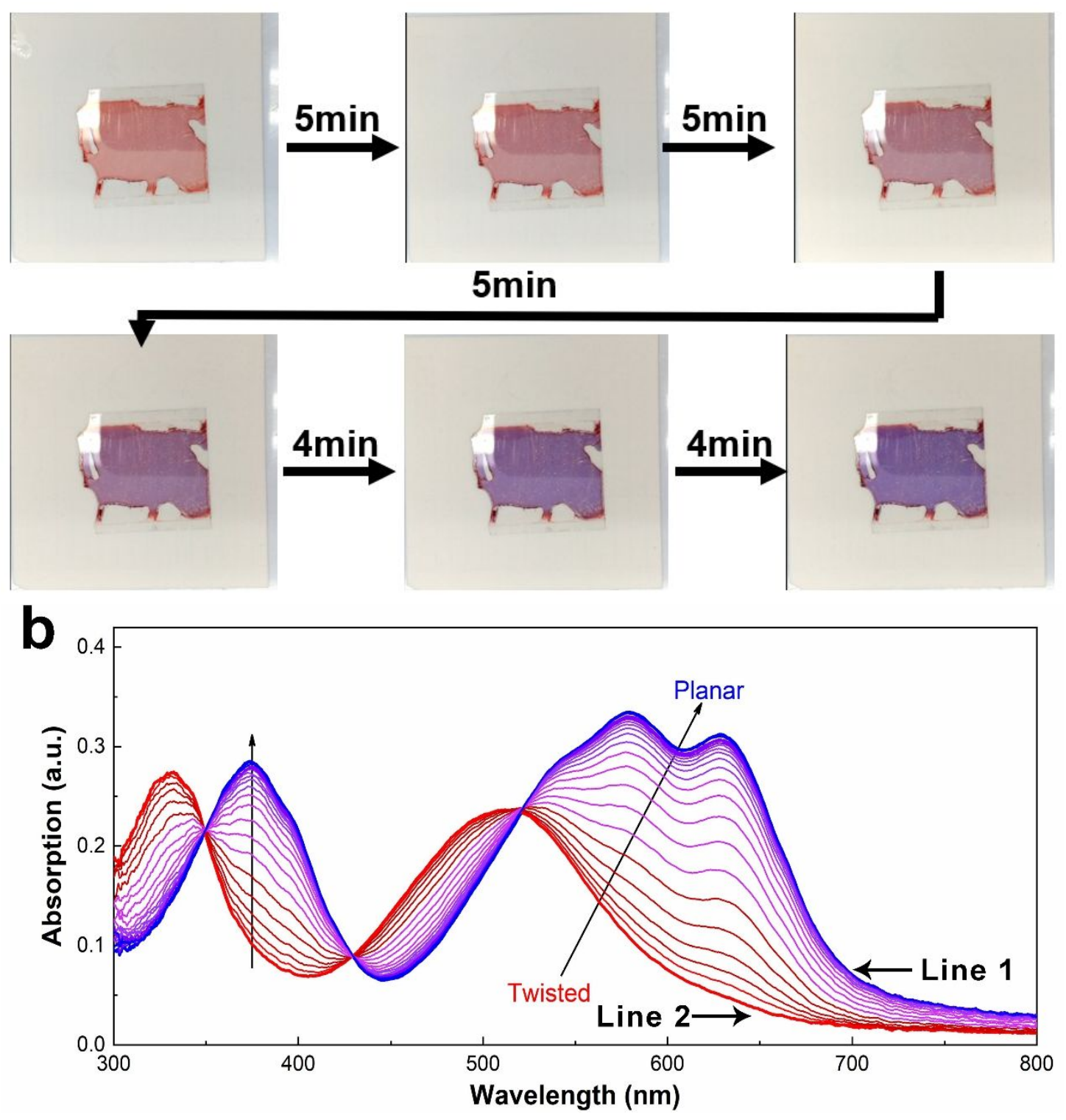

Figure S1 a) The topological transition of TT film from twisted state (red) to planar state (dark blue) after heating under $125^{\circ} \mathrm{C}$ for 3 seconds. b) The absorption spectra during the transition of the film. Following the spin-coating of TT molecule in $\mathrm{CHCl}_{3}$ solvent, it yields a red film, and slowly turns to dark blue as shown in line 1 in the spectra graph. Thereafter, the film was heated under $125^{\circ} \mathrm{C}$ for $5 \mathrm{~s}$, the molecule then becomes red again where the absorptance was shown by line 2 in the figure. Then, the scans from $800 \mathrm{~nm}$ to $300 \mathrm{~nm}$ were conducted every 70 seconds, and the absorption curve slowly shifts to right. Hence, the color of the film changes back to dark blue after 
around 23 min (20 times scans) at room temperature, and the absorption curve overlaps with line 1 again.

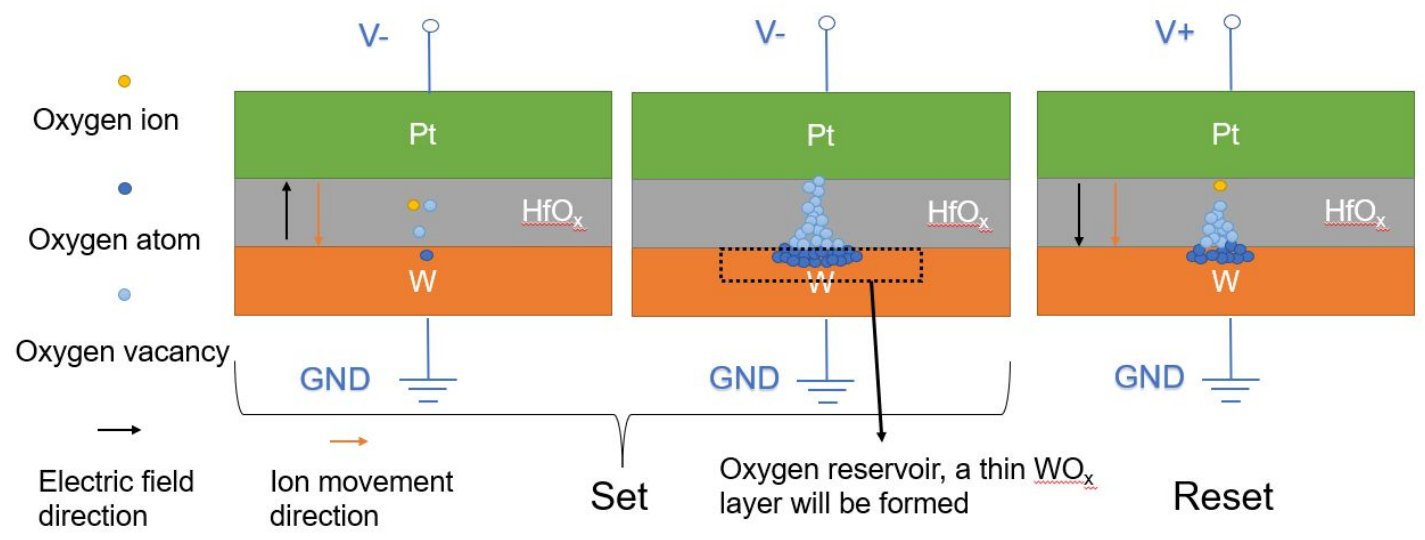

Figure S2 Schematic illustration on set/reset process of Pt/HfOx/W ReRAM stack

a

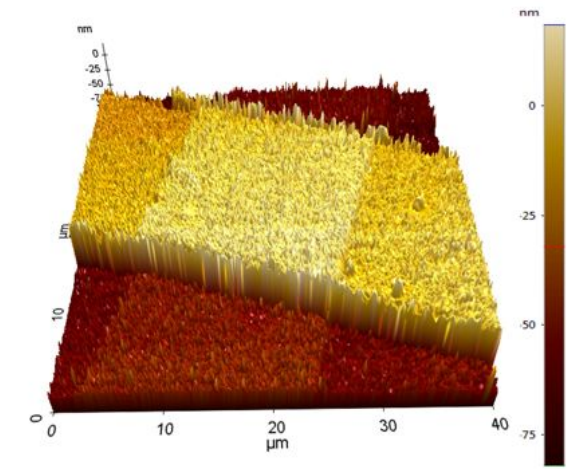

C

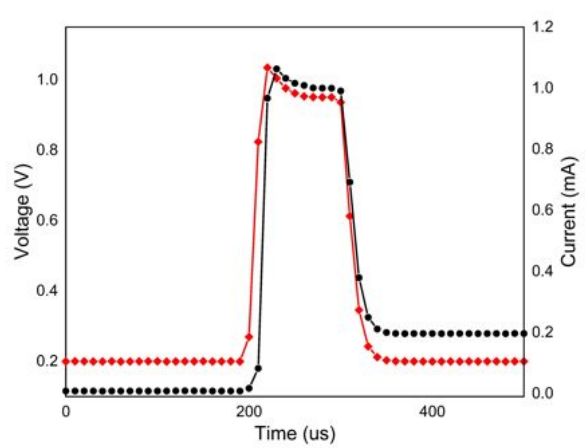

b

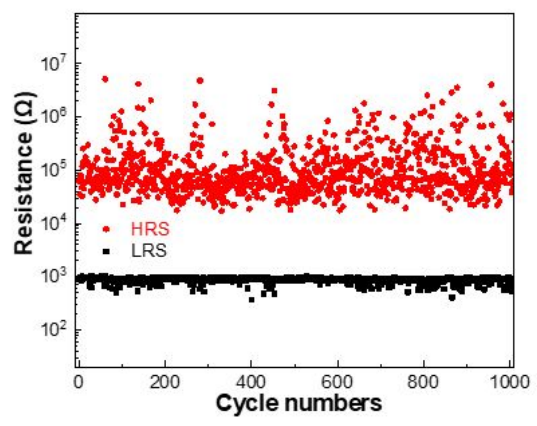

d

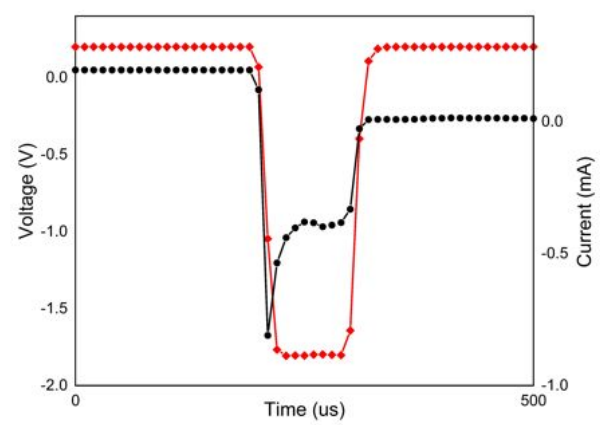

Figure S3 a) Heightmap of ReRAM device area characterized by AFM. b) The 1024 cycles DC endurance data for W(10nm)/HfOx(10nm)/Pt(60nm) ReRAM stack with a $0.2 \mathrm{~V}$ read voltage for both HRS and LRS in each cycle. c) The transient response during set process for fabricated ReRAM with $100 \mu$ s pulse width and 1.0V pulse amplitude (red line). The current response of ReRAM is less than $20 \mu$ s (Blackline). d) The 
transient response during reset process for fabricated ReRAM with $100 \mu$ s pulse width and $-1.8 \mathrm{~V}$ pulse amplitude (red line). The current response of ReRAM is less than $20 \mu \mathrm{s}$ (Blackline)

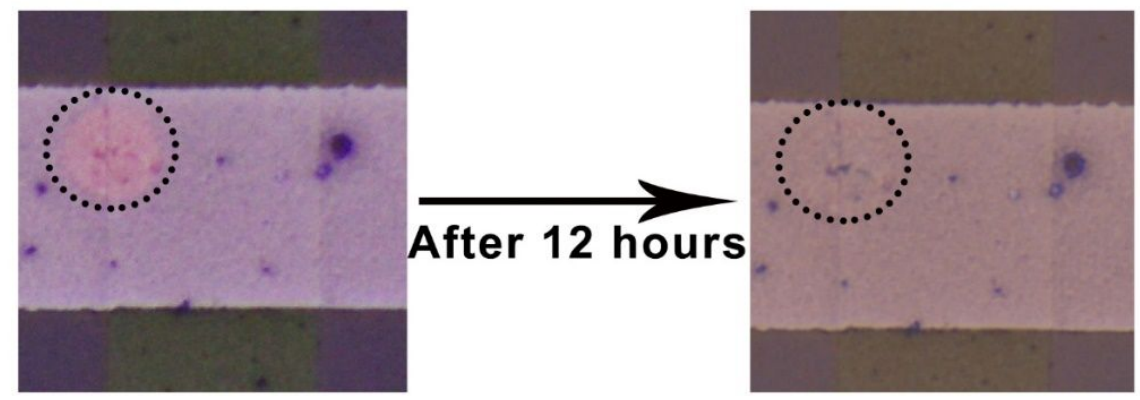

Figure S4 One of the color-changing spots that induced by the resistive switching and recovered after 12 hours.

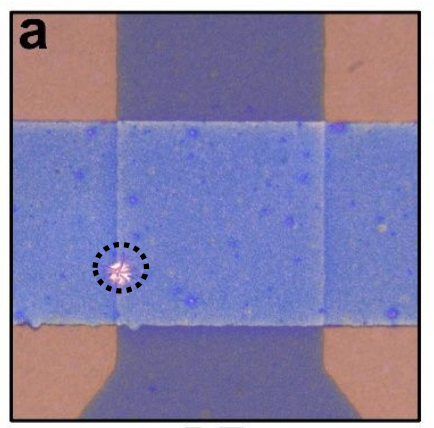

BE

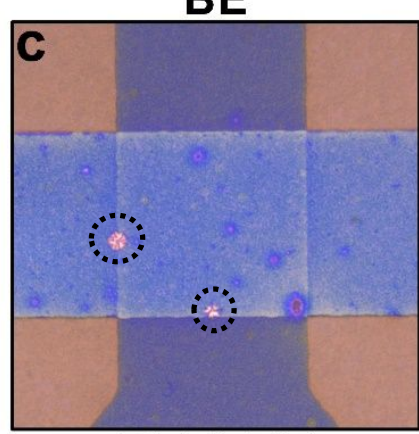

BE \& TE

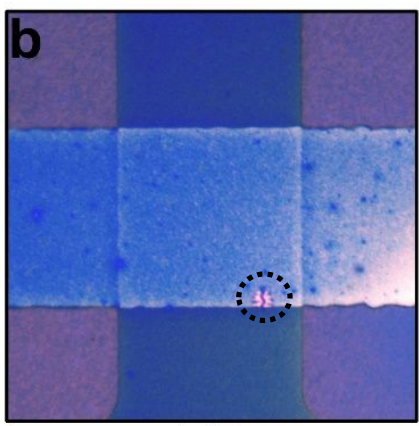

TE

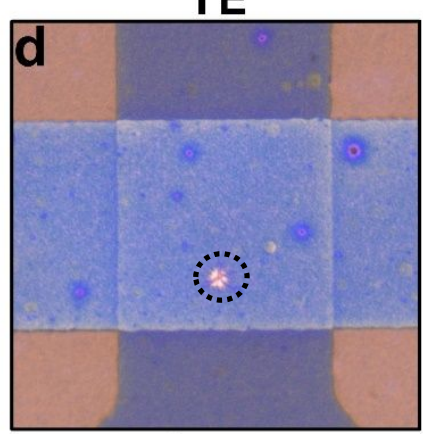

Center

Figure S5 Optical images of 'color-changed' ReRAM devices that have filaments formation at BE edge (a), TE edge (b), multiple filaments (c), and center (d), while in most ReRAM devices, a single dominant filament will be formed. 
a

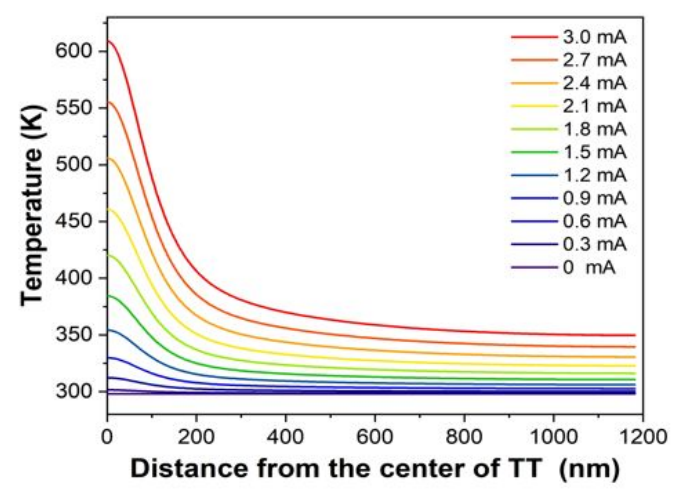

C

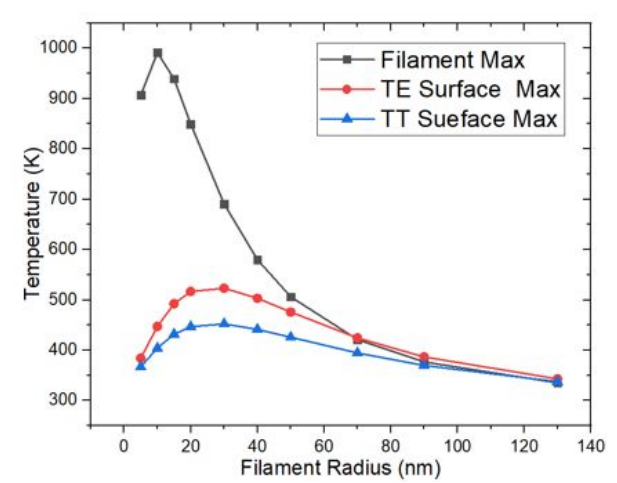

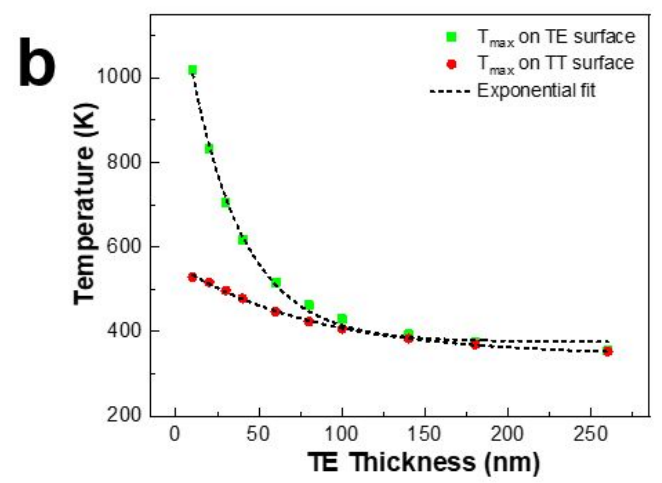

d

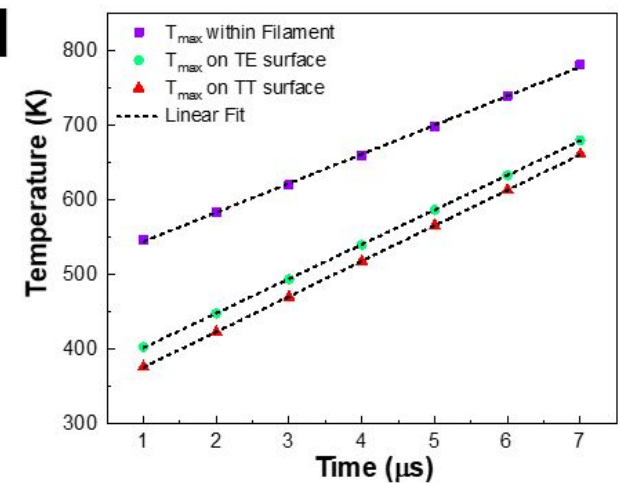

Figure S6 a) The temperature distribution on TT surface with varying applied current values from 0 to $3 \mathrm{~mA}$. b) The maximum temperature value and their exponential fittings of TE surface (green) and TT surface (red) with varying TE thickness. c) The maximum temperature value in filament, on TE surface, and TT surface with varying filament radius from $5 \mathrm{~nm}$ to $130 \mathrm{~nm}$. d) The temperature increment in filament, on TE surface, and TT surface with time, which the boundaries of the model were set to be thermally insulating.

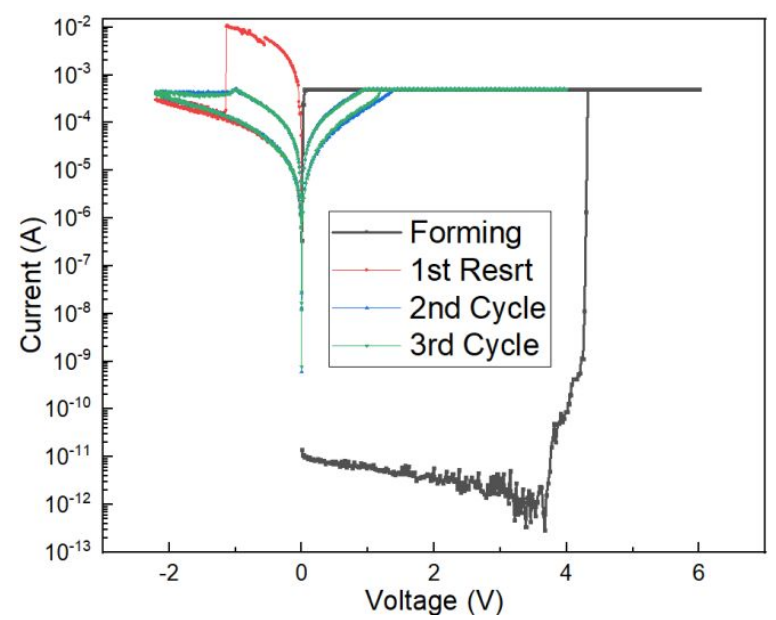


Figure S7 The I-V curves for ITO (substrate) /HfOx (12nm)/W (the head of the tip) ReRAM stack, where the micro-tip could control the filament formation inside the oxide layer.

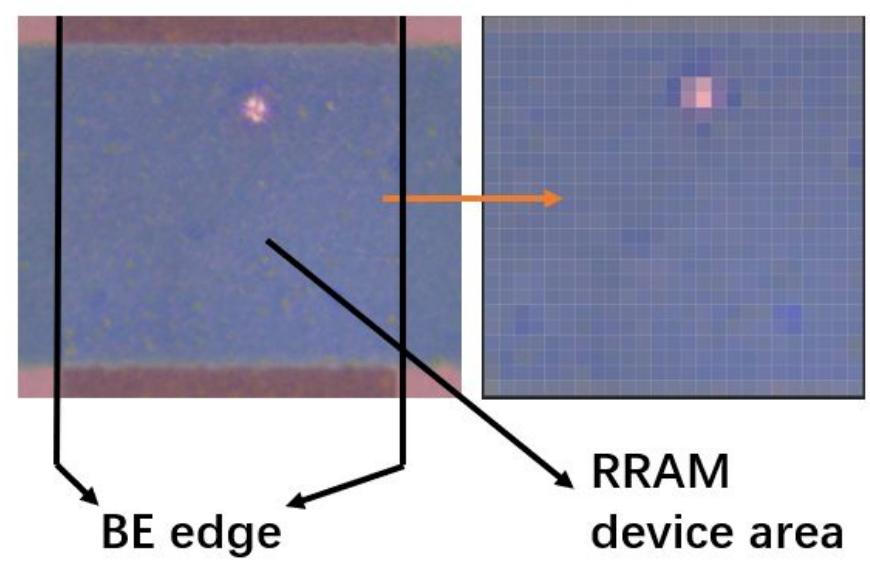

Figure S8 The demonstration on the way to get the filament coordinate in statistical studies. The ReRAM device area was divided into small pixels, where each of them represents $2 \mu \mathrm{m} \times 2 \mu \mathrm{m}$, hence, the filaments were defined at BE edge if the color changing pixel located inside the first and the last column, otherwise, they were defined at middle.

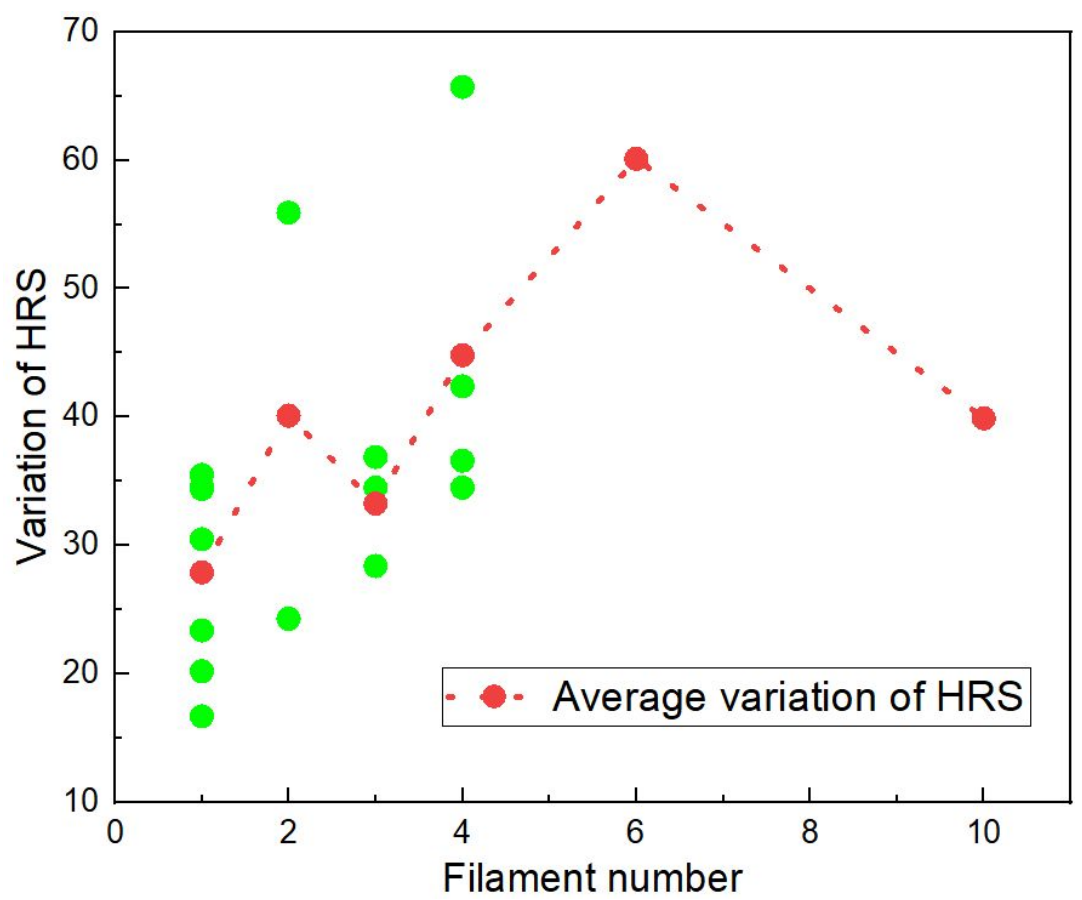


Figure S9 The variations of HRS in 32DC cycles for 18 ReRAM devices, and its relationship with the $\mathrm{CF}$ number.

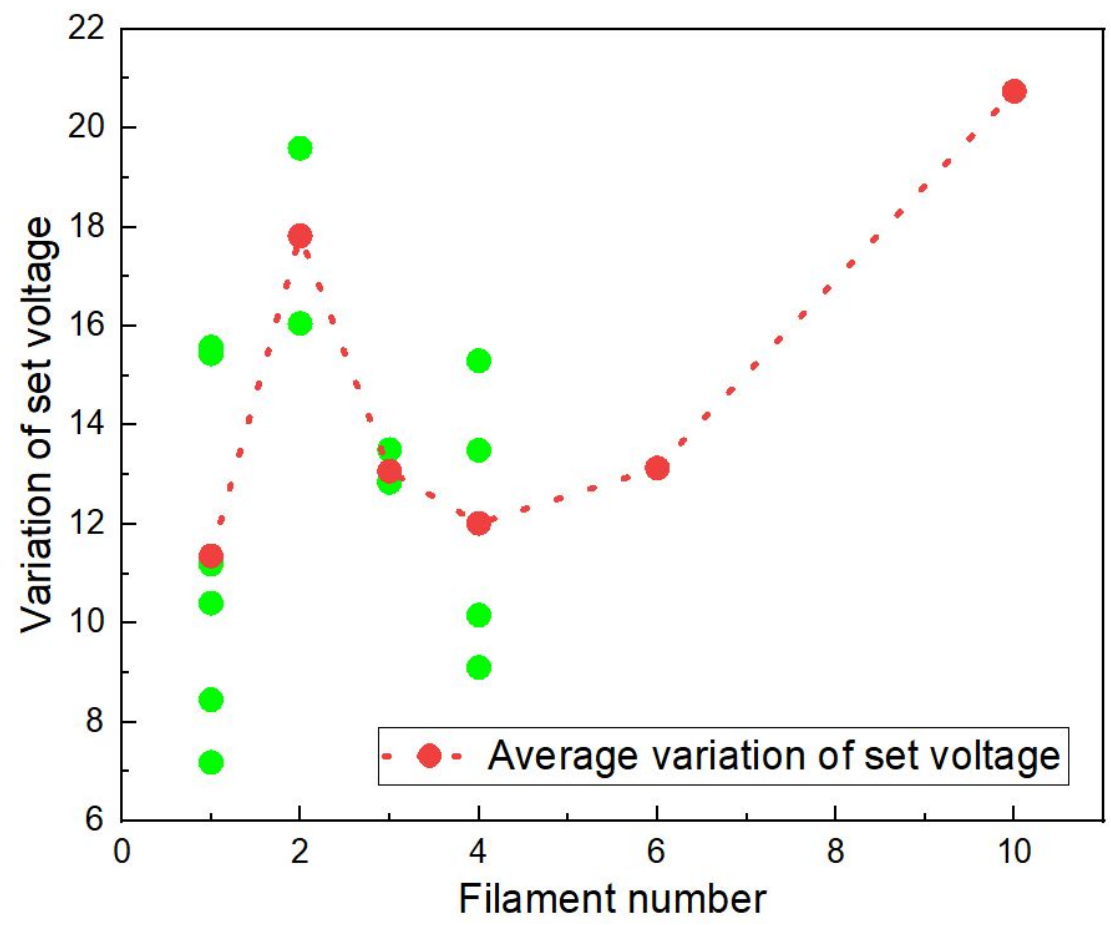

Figure S10 The variations of set voltage in 32DC cycles for 18 ReRAM devices, and its relationship with the CF number.

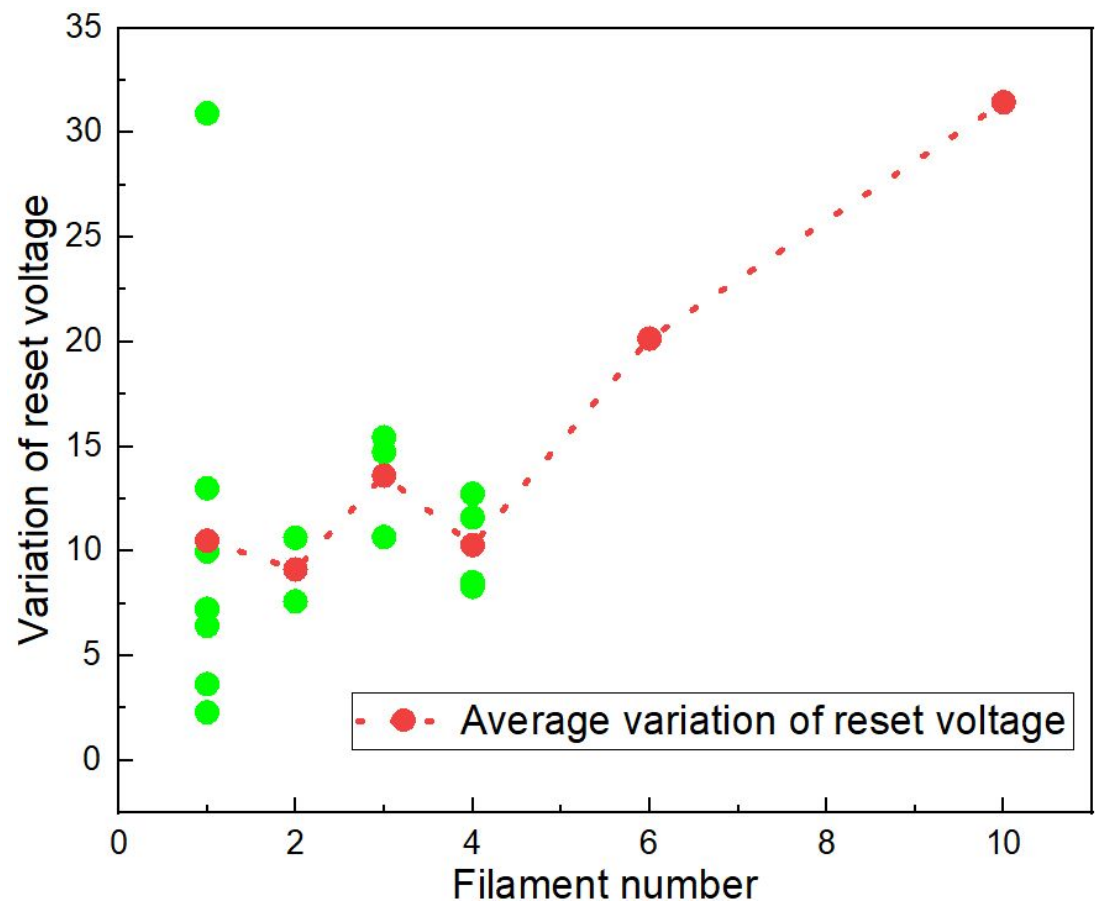


Figure S11 The variations of reset voltage in 32DC cycles for 18 ReRAM devices, and its relationship with the CF number.

\section{Note 1: Parameters in COMSOL simulation}

Since Si has a very high thermal conductivity compared to $\mathrm{SiO}$, and the $\mathrm{Si}$ substrate is large in thickness, the temperature at bottom of $\mathrm{SiO} 2$ layer was set to room temperature (297K).28 Since the air has a very low thermal conductivity $\left(\sim 0.02 \mathrm{~W} / \mathrm{m}^{*} \mathrm{~K}\right)$, and the upper TT film was ultrathin, the upper and right side of the model was set to be thermally insulating. Heat transfer, electrical current, and electrical circuit modules were used during the simulation. A stationary study was conducted to get the temperature information inside the model.

For Tungsten bottom electrode, $\mathrm{HfO}_{2}$ dielectric, and $\mathrm{Pt}$ top electrode, the material parameters are from the build-in library inside COMSOL. For the parameters of $\mathrm{HfO}_{2-}$ ${ }_{x}$ conductive filament and TT molecule layer, the parameters of them were summarized as following table $\mathrm{S}^{1 .}{ }^{1-3}$ (TT is an organic semiconducting small molecule. Hence, using another common organic semiconducting material P3HT to replace TT because of the high similarity.)

\begin{tabular}{|l|c|c|}
\hline \multicolumn{1}{|c|}{ Parameter description } & HfO $_{\mathrm{x}}$ filament & T molecule \\
\hline Relative permittivity & -1000000 & 3.02 \\
\hline Thermal conductivity $(\mathrm{W} / \mathrm{m} \star \mathrm{K})$ & 0.65 & 0.2 \\
\hline Specific heat capacity $(\mathrm{J} / \mathrm{kg} \star \mathrm{K})$ & 140 & 1320 \\
\hline Density $\left(\mathrm{kg} / \mathrm{m}^{3}\right)$ & 12000 & 1100 \\
\hline Electrical conductivity $(\mathrm{S} / \mathrm{m})$ & 7000 & 12.7 \\
\hline
\end{tabular}

Table S1. The parameters of $\mathrm{HfO}_{\mathrm{x}}$ conductive filament and TT molecule layer used in COMSOL simulation.

\section{Note 2: Experimental section}


ReRAM device fabrication: The 4-inch silicon wafer covered with $300 \mathrm{~nm} \mathrm{SiO}_{2}$ was cut into $1.5 \mathrm{~cm}$ by $1.5 \mathrm{~cm}$ and cleaned using acetone and IPA sequentially in the ultrasonic bath for 5 min each. AZ5214B photoresist was spin-coated on the cleaned substrate at $6000 \mathrm{rpm}$ for $60 \mathrm{~s}$, followed by soft baking at $110{ }^{\circ} \mathrm{C}$ on a hot plate for 2 min. After the UV exposure, substrates were developed using AZ531B, which is diluted with four parts of deionized (DI) water, for $20 \mathrm{~s}$. Thin-film deposition of the metal and insulator layers were performed in an AJA-8 gun sputtering system with a base pressure of better than $1 \times 10^{\wedge}(-8) \quad$ Torr. After deposition, the photoresist is lifted-off using acetone and IPA in ultrasonic for 10min each, and the first layer (BE) is completed. The processes of lithography, sputtering, and lift-off were repeated for the dielectric and TE layers. The sputter processes are maintained at 2 mtorr with $20 \mathrm{sccm}$ Ar at room temperature. $50 \mathrm{~W}$ DC was used for metal materials deposition while $50 \mathrm{~W}$ RF was used for oxide materials deposition.

Electrical characterization: The electrical characterizations during the study were conducted by Keysight Precision Source Measure Unit (B2900A Series) and a probe station (Karl Suss PM5).

Preparation of TT film: TT molecule is synthesized as previously reported. ${ }^{32,33}$ The TT molecule was dissolved in chloroform solvent to form a $5 \mathrm{mg} / \mathrm{ml}$ solution, which is followed by spin-coating on the fabricated ReRAM device at $3000 \mathrm{rpm}$ for $30 \mathrm{~s}$ in a nitrogen-filled glove box. For the UV-vis sample, $10 \mathrm{mg} / \mathrm{ml}$ TT solution was prepared and spin-coated on a coverslip at $1000 \mathrm{rpm}$ for $30 \mathrm{~s}$.

TEM sample preparation: The TEM lamella prior to TEM observation were prepared using a Zeiss Crossbeam 540 FIB-SEM. The devices were initially protected with electron and ion beam deposited Pt films inside the FIB, followed by milling from a coarse current of $15 \mathrm{nA}$ at $30 \mathrm{kV}$ to a final low $\mathrm{kV}$ polishing at $5 \mathrm{kV}, 10 \mathrm{pA}$, with decreasing current and voltage during the milling process. The bright-field TEM images of the full device were analyzed in JOEL TEM 2010 HR.

\section{Note 3: Lamella preparation:}


The sample was milled inside a Zeiss Crossbeam 540 FIB-SEM to reach electron transparency using $\mathrm{Ga}+$ ion source operating at $30 \mathrm{kV}$. Final polishing was done at 5 $\mathrm{kV}$ on the sample to remove $\mathrm{Ga}+$ ion contamination during the milling. Beam currents were varied from $15 \mathrm{nA}$ during initial coarse milling to $100 \mathrm{pA}$ during the final thinning. Since the FIB gun was oriented at 54o with respect to the electron gun, the stage was tilted to 54o during the milling process and the Pt deposition process. Hence, the specimen surface was oriented perpendicular to the FIB gun.

Before focus ion beam (FIB) Pt deposition, $1 \mu \mathrm{m}$ layer of Pt was first deposited on a rectangular area of $2 \mu \mathrm{m}$ wide and $10 \mu \mathrm{m}$ long using electron beam at $2 \mathrm{nA}$. The stage was kept at $0 \mathrm{o}$ so that it is perpendicular to the electron gun. This step is essential in ensuring that the area of interest is protected from the ion beam damage during the milling process. Subsequently, the stage was tilted to 54o. Another $1 \mu \mathrm{m}$ layer of $\mathrm{Pt}$ was deposited on top of the electron beam deposited Pt using FIB Pt deposition at 30 $\mathrm{kV}, 300 \mathrm{pA}$. The final Pt layer was $2 \mu \mathrm{m}$ thick.

While keeping the stage tilted at 54o, coarse milling at $15 \mathrm{nA}$ was done to create two trapezoid-shaped trenches of $8 \mu \mathrm{m}$ deep and $15 \mu \mathrm{m}$ high (one positioned $1 \mu \mathrm{m}$ on top and another $1 \mu \mathrm{m}$ below the area with Pt deposition). The lamella created after this step was $4 \mu \mathrm{m}$ thick and $10 \mu \mathrm{m}$ wide. Since subsequent FIB milling can cause material redeposition on two sides of the lamella surface, posing a problem for subsequent imaging. Hence, polishing was done on both sides of lamella at $30 \mathrm{kV} 3 \mathrm{nA}$ so that the final lamella was $2 \mu \mathrm{m}$ thick and $10 \mu \mathrm{m}$ wide. When polishing the lamella bottom surface and upper surface, the stage was tilted to 560 and 540 respectively.

The stage was then tilted back to 0o, allowing the FIB to image the surface of the lamella. A U-shaped cut was then made on the lamella surface while leaving the small edge attached to the bulk specimen for anchoring during the transfer process. Afterwards, the lamella was attached to a needle via $600 \mathrm{~nm}$ layer of Pt deposited across the needle and the lamella surface. The deposition was done at $30 \mathrm{kV}, 300 \mathrm{pA}$. After removing the edge of the lamella attached to the bulk specimen, the lamella was then glued side-way on one post of a copper Omniprobe TEM grid for further thinning. 
During the transfer, the lamella was occasionally imaged using secondary electrons at $5 \mathrm{kV}, 50 \mathrm{pA}$ and FIB at $30 \mathrm{kV}, 5 \mathrm{pA}$. To reduce any possible damage caused by ion beam exposure, reduced window was used to expose only the edge of the lamella for imaging.

For further thinning process, the grid with the attached lamella was tilted to 54 the lamella was first thinned at $30 \mathrm{kV} 700 \mathrm{pA}$. When the thickness was roughly $1 \mu \mathrm{m}, \mathrm{FIB}$ current of $300 \mathrm{pA}$ at $30 \mathrm{kV}$ was used to further thin down the lamella. Afterwards, when the lamella was thinner than $500 \mathrm{~nm}$, FIB current was changed to $100 \mathrm{pA}$ at $30 \mathrm{kV}$ to further thin the lamella to below $200 \mathrm{~nm}$. Subsequently, the film was polished at $5 \mathrm{kV}$ 10pA. Finally, the lamella attached on the grid was then loaded on a single tilt holder and analyzed in JOEL TEM 2010 HR.

\section{Reference.}

1. Kim, S.; Kim, S.-J.; Kim, K. M.; Lee, S. R.; Chang, M.; Cho, E.; Kim, Y.-B.; Kim, C. J.; Chung, U.-I.; Yoo, I.-K., Physical electro-thermal model of resistive switching in bi-layered resistance-change memory. Scientific reports 2013, 3 (1), 1-6.

2. Chen, S. Dielectric constant measurement of P3HT, polystyrene, and polyethylene. Faculty of Science and Engineering, 2017.

3. Niraula, D.; Karpov, V., Comprehensive numerical modeling of filamentary RRAM devices including voltage ramp-rate and cycle-to-cycle variations. Journal of Applied Physics 2018, 124 (17), 174502. 